\title{
Technology Challenges in Small UAV Development
}

\author{
Michael J. Logan, P.E.* and Thomas L. Vranas ${ }^{\dagger}$ \\ NASA Langley Research Center, Hampton, Virginia, 23681 \\ Dr. Mark Motter ${ }^{\ddagger}$ and Dr. Qamar Shams ${ }^{\S}$ \\ NASA Langley Research Center, Hampton, Virginia, 23681 \\ Dion S. Pollock ${ }^{* *}$ \\ United States Army
}

\begin{abstract}
Development of highly capable small UAVs present unique challenges for technology protagonists. Size constraints, the desire for ultra low cost and/or disposable platforms, lack of capable design and analysis tools, and unique mission requirements all add to the level of difficulty in creating state-of-the-art small UAVs. This paper presents the results of several small UAV developments, the difficulties encountered, and proposes a list of technology shortfalls that need to be addressed.
\end{abstract}

\section{Nomenclature}

$P \quad=$ Power required for flight

$D \quad=$ Aircraft Drag

$V \quad=$ Aircraft Velocity

$P_{s} \quad=$ Specific excess power

COTS $=$ Commercial Off-the-Shelf

VTOL $=$ Vertical Takeoff or Landing

EMI = Electro-magnetic Interference

RFI $=$ Radio Frequency Interference

\section{Introduction}

$\mathrm{T}$

he development of small, highly capable unmanned aerial vehicles (UAVs) requires consideration of the platform, payload, propulsion, and overall mission as an integrated system. As such, the analyses of each of the elements of the system must be able to be considered concurrently. At issue is whether each of these analysis elements have the necessary accuracy and completeness to be used to optimize the final vehicle configuration. Each of the analysis elements and their corresponding problems will be described below. An example from the development activities of the NASA Langley Small Unmanned Aerial Vehicle Laboratory (SUAVELab) will be used to demonstrate each issue.

\section{Platform Design Issues}

\footnotetext{
${ }^{*}$ Head, Small Unmanned Aerial Vehicle Laboratory (SUAVELab), MS238 NASA Langley Research Center, Member AIAA.

${ }^{\dagger}$ Lead Engineering Technician, SUAVELab.

${ }^{\ddagger}$ Research Engineer, Electronics Systems Branch, NASA Langley research Center, member AIAA.

${ }^{\S}$ Research Engineer, Aeronautics Systems Engineering Branch, NASA Langley Research Center.

** Sergeant First Class, United States Army, assigned to Army Research Laboratory, member AIAA. 
Many of the UAVs being investigated by the SUAVELab involve lightweight electric powered air vehicles. The design process for this class of vehicles is dissimilar to that of larger, fuel-burning air vehicles. In fueled vehicles, a takeoff weight is assumed, an empty weight is estimated based on the takeoff weight, the mission is "flown" computationally, and the weight at end-of-mission is compared to the previously estimated empty weight. A "fuel balance" is performed where weight is added or subtracted from the takeoff weight and the process iterates until a convergence occurs. Since the electric vehicles do not change weight, the primary architecture of the historical design synthesis methodologies are non-useful. An alternate design process was developed for these small, electric powered vehicles and is shown in Figure1.

In the alternate design process, the first step is to assume a takeoff weight. This takeoff weight helps to bound the expected size of the vehicle. In the case of a man-portable UAV, this weight must be limited to what an individual can carry without an undue burden (<2-3lb. maximum). For a hand-launched $\mathrm{UAV}$, the weight must be light enough for an average individual to accelerate the vehicle to its minimum flight speed $(<10-12 \mathrm{lb}$. maximum). Once a target weight has been identified, an estimate or assumption is made as to the achievable lift vs. drag ratio (L/D) at the target mission airspeed. The $\mathrm{L} / \mathrm{D}$ ratio can sometimes be estimated from the performance of similar vehicles when such similarity exists. However, there are few computational methods valid for this class of vehicles owing to limitations in low Reynold's number aerodynamics prediction and limitations in the types of configurations for which empirical flight test or wind tunnel data is available. Given a weight and L/D ratio, the amount of power required for level flight can be assessed for various airspeeds by using the basic equation:

$$
\mathrm{P}=\mathrm{DV}
$$

Note that the preceding equation can be used for both straight and level flight and for any flight constraints, such as the power

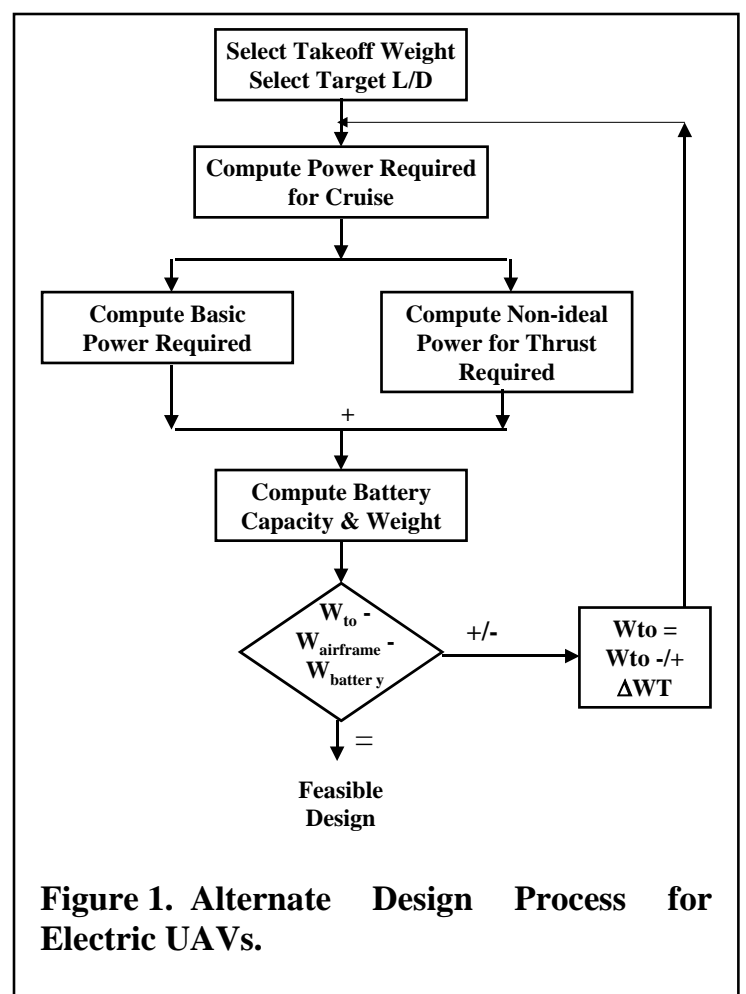
required to turn. For most of the small reconnaissance UAVs, a 2-g turn should be considered a minimum power requirement. Once the basic power required for level flight in cruise is established, a nominal battery size can be established by computing the capacity required for the mission. In addition, a secondary analysis must be run to determine the efficiency with which the propulsion system can produce thrust. This efficiency loss must be added back into the "ideal" current usage to determine a realistic battery size. Once the battery capacity and subsequent weight is computed, it can be compared to the takeoff weight to determine if there is sufficient weight margin remaining for airframe, systems, and payload. In a fashion similar to the fueled aircraft, the process is repeated until a convergence occurs, assuming there is a feasible solution. There can be problematic elements in this analysis, however.

At present, there are few computational methods to predict component weights for this class of vehicle. Generally, the components are few enough to use a spreadsheet for simply listing the component weights. Airframe weight is problematic in that although CAD data files can be used to determine some of the weight based on material volume and density, this represents only $50-75 \%$ of the actual airframe weight, depending on construction technique used. This is a particularly acute problem for the aircraft in Figure 2 which is designed as a long endurance electric UAV.

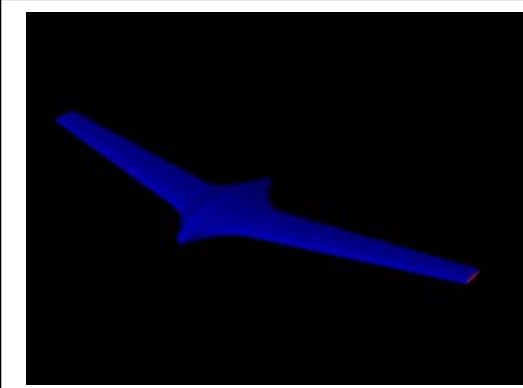

Figure 2. Long-endurance electric UAV design. 


\section{Propulsion System Analysis and Selection Issues}

Selecting the "optimum" propulsion system (i.e. motor, gearbox, propeller, motor controller, and battery) is crucial for an electric UAV. Additionally, the more stringent the endurance requirement, the more tailored the propulsion system must be to achieve the mission. However, currently available methods for electric propulsion analysis and optimization greatly lag their fueled counterparts. Table 1 shows measured performance data for the propulsion system of the VTOL UAV shown in Figure 3. The test unit measured the electric motor, gearbox, and propeller system performance using a fixed $11.1 \mathrm{~V}$ input provided by a power supply. Predicted maximum thrust of the system using a 3-cell lithium-ion-polymer battery, nominally $11.1 \mathrm{~V}$, was approximately $10 \%$ higher than measured actual. When an actual 3-cell battery pack was used, the measured RPM was 6250 , or approximately $16 \%$ less than actual, $25 \%$ less than predicted. Using a 4-cell li-poly pack only added approximately $10 \%$ net thrust, far less than predicted. Clearly this indicates the

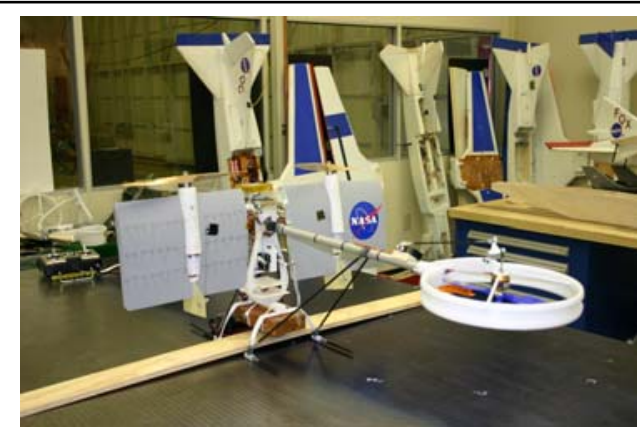

Figure 3. VTOL UAV prototype. commonly available analysis methods are not accounting for some type of basic phenomena occurring in the system.

Table 1. Measured performance data, Hacker B40-12L, Zinger 12x8 propeller.

\begin{tabular}{|c|c|r|r|r|r|r|r|r|r|}
\hline Throttle Setting & Pulse Width (msec) & \multicolumn{1}{|c|}{ Volts } & Amps & \multicolumn{1}{c|}{$\begin{array}{c}\text { Power } \\
\text { Watts }\end{array}$} & Deg F & $\begin{array}{r}\text { Torque } \\
\text { inch lbs }\end{array}$ & k RPM & \multicolumn{1}{c|}{$\begin{array}{r}\text { Thrust } \\
\text { lbs }\end{array}$} & $\begin{array}{r}\text { Air Vel } \\
\text { kft/min }\end{array}$ \\
\hline 1 & 1.15 & 11.089 & 0.109 & 1.205 & 98.935 & 0 & 0.036 & 0.004 & 0.007 \\
2 & 1.18 & 11.091 & 0.108 & 1.203 & 98.837 & 0 & 0.036 & 0.004 & 0.007 \\
3 & 1.2 & 11.092 & 0.108 & 1.2 & 98.769 & 0 & 0.034 & 0.003 & 0.007 \\
4 & 1.23 & 11.089 & 1.259 & 13.956 & 98.272 & 0 & 1.736 & 0.199 & 0.329 \\
5 & 1.27 & 11.089 & 2.097 & 23.252 & 97.164 & 0.265 & 2.228 & 0.325 & 1.041 \\
6 & 1.31 & 11.091 & 3.086 & 34.231 & 96.066 & 0.642 & 2.649 & 0.461 & 1.407 \\
7 & 1.34 & 11.092 & 4.18 & 46.362 & 94.936 & 1.059 & 3.019 & 0.602 & 1.676 \\
8 & 1.37 & 11.091 & 5.383 & 59.701 & 93.948 & 1.517 & 3.361 & 0.754 & 1.852 \\
9 & 1.4 & 11.089 & 6.629 & 73.511 & 93.167 & 1.992 & 3.663 & 0.907 & 2.032 \\
10 & 1.44 & 11.09 & 8.153 & 90.423 & 92.444 & 2.572 & 3.988 & 1.093 & 2.256 \\
11 & 1.47 & 11.091 & 9.593 & 106.401 & 91.895 & 3.12 & 4.262 & 1.254 & 2.452 \\
12 & 1.51 & 11.091 & 11.351 & 125.898 & 91.265 & 3.79 & 4.569 & 1.423 & 2.633 \\
13 & 1.54 & 11.089 & 12.788 & 141.808 & 90.742 & 4.337 & 4.79 & 1.581 & 2.84 \\
14 & 1.57 & 11.09 & 15.287 & 169.538 & 90.091 & 5.289 & 5.123 & 1.802 & 3.039 \\
15 & 1.6 & 11.091 & 17.67 & 195.966 & 90.091 & 6.196 & 5.447 & 2 & 3.332 \\
16 & 1.63 & 11.091 & 20.788 & 230.563 & 89.868 & 7.384 & 5.795 & 2.269 & 3.574 \\
17 & 1.67 & 11.09 & 24.29 & 269.374 & 89.961 & 8.717 & 6.137 & 2.566 & 3.664 \\
18 & 1.69 & 11.09 & 28.219 & 312.936 & 90.059 & 10.214 & 6.459 & 2.856 & 4.044 \\
19 & 1.73 & 11.092 & 32.302 & 358.284 & 90.455 & 11.769 & 6.792 & 3.137 & 4.191 \\
20 & 1.76 & 11.091 & 37.139 & 411.911 & 91.035 & 13.611 & 7.097 & 3.51 & 4.444 \\
21 & 1.79 & 11.09 & 42.934 & 476.13 & 91.755 & 15.818 & 7.458 & 3.842 & 4.759 \\
22 & 1.82 & 11.09 & 43.001 & 476.898 & 92.145 & 15.843 & 7.442 & 3.857 & 4.796 \\
\hline
\end{tabular}

Beyond issues in propulsion performance prediction, there is the issue of system "sizing". In a more traditional aircraft sizing, a "rubber" engine can be used to determine the optimum cycle parameters and constraints such as takeoff field length, $\mathrm{P}_{\mathrm{s}}$, and others can be used to determine the overall thrust-to-weight required. In the case of small electric UAVs, the thrust required for takeoff is large relative to the thrust at cruise. Further, the combination of motor, gearbox, and propeller that optimizes static thrust for takeoff is dissimilar to that which minimizes current draw in cruise. Table 2 shows the thrust and current draw for a set of motors under consideration. Note that the last item in the table is a motor, prop, and gearbox optimized for cruise which is wholly inadequate to provide the necessary static thrust for hand launch. A useful invention for this class of vehicles would be an electric motor analogous to a variable cycle engine in the turbine world.

Table 2. Electric motor estimated data.

\begin{tabular}{|l|r|r|r|l|}
\hline \multicolumn{1}{|c|}{ Motor } & $\begin{array}{c}\text { Takeoff } \\
\text { Thrust } \\
\text { (oz.) }\end{array}$ & $\begin{array}{c}\text { Cruise } \\
\text { Current } \\
\text { Draw (Amps) }\end{array}$ & $\begin{array}{c}\text { Cruise } \\
\text { Power } \\
\text { (Watts) }\end{array}$ & $\begin{array}{l}\text { Vmax } \\
\text { (mph) }\end{array}$ \\
\hline B50-9XL+6.67:1 gearbox & 172 & 13.6 & 220 & 70 \\
Actro 32-4 & 170 & 11.5 & 134 & 90 \\
PJS 5000 & 176 & 15.5 & 183 & 92 \\
& 73 & 5.5 & 75 & 87 \\
\hline B40-181+4.4:1 gearbox & 73 & & \\
\hline
\end{tabular}




\section{Subsystems and Integration Issues}

When developing prototype UAVs, especially those whose purpose is to be "low cost" the traditional approach are to use as many COTS components as possible. Use of these components is not without risk, however. Table 3 shows component failure rates for several types of commonly used components. Note that these failure rates include both electronic failures (where the components fail to function or function erratically) as well as mechanical failures in the gear train or control arms. In addition to component failures, there is a significant risk of incompatibility using COTS components in even moderately complex situations. For example, Figure 4 shows a small, man portable electric UAV. The internal control system of this vehicle is shown in Figure 5. For this configuration, over 30 combinations of different vendor's electronic speed control, gyro, electronic signal mixer, and servos were tested. Over $60 \%$ of the configurations tested had some form of problem consisting of either erratic behavior, jitter, control reset, overheating, or system instability over time. Improvements in avionics reliability, stability, and compatibility are a clear need, particularly in low-cost applications.

Table 3. Component failure rates.

\begin{tabular}{|l|r|}
\hline Item & In-use failure rate \\
\hline Servos, analog & $20 \%$ \\
Servos, digital & $5 \%$ \\
Electronic speed controller & $30 \%$ \\
Mixers & $12 \%$ \\
Gyros (all types) & $8 \%$ \\
Motors (all types) & $10 \%$ \\
\hline
\end{tabular}

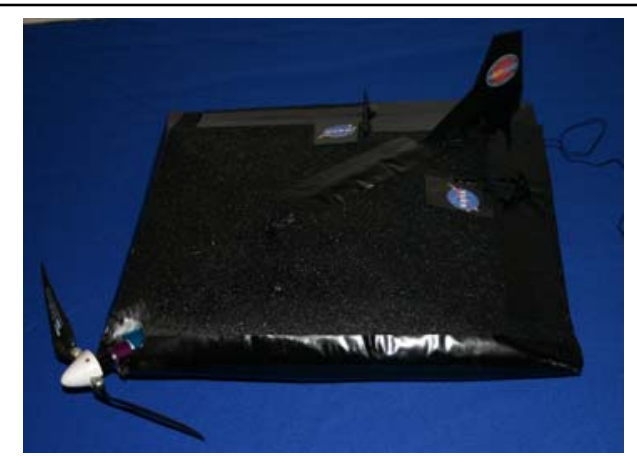

Figure 4. "Satchel Plane” small UAV.

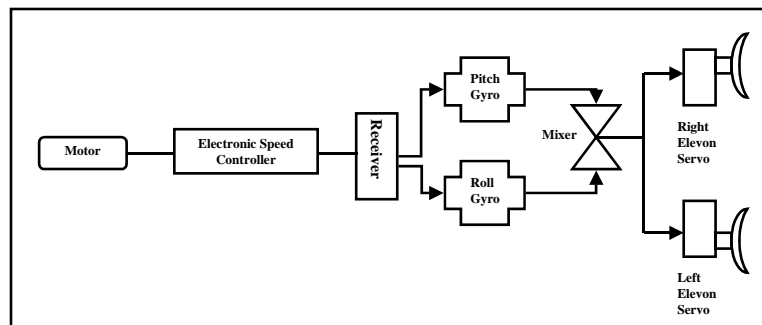

Figure 5. Electronics arrangement of small UAV.

In addition to component reliability, other avionics related issues can manifest themselves during development. For example, in the case of smaller UAVs where the avionics are densely packed in close proximity to each other and the propulsion system, EMI and RFI issues can occur. Figure 6 shows the interior of an autonomous UAV. In this instance, protective shielding was used throughout the center and forward bays because of the power levels of the video and command link radio power levels. However, the accidental replacement of a composite pushrod with a metal one created a direct transfer of radio noise to the forward servo and, in turn, to the rest of the avionics. Further testing revealed that many commercially available servos are particularly sensitive to spread-spectrum 900Mhz RF emissions.

Sensor and processing technology is another area where technology improvements could have a potentially significant

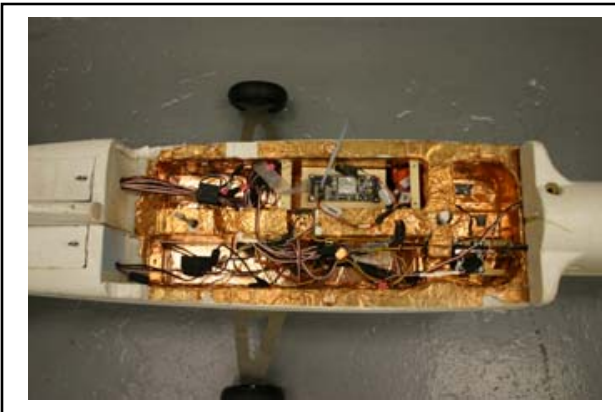

Figure 6. Interior bay of NASA modified Army FQM-117B UAV. impact. Most commercially available auto-navigation units are more expensive than a low-cost application can reasonably accommodate, thus precluding their use. Capable units that have both adequate sensors and computational throughput for advanced algorithms should be based, if possible, on commodity hardware to the extent such hardware can fit the application. Sensor technology that can sense drift in a VTOL hover without becoming saturated by vibration noise is another area where research is needed. Low-cost, low-installation penalty 
collision avoidance is another technology need. Transition from analog video downlink to video and command link via commodity digital broadband communications could also provide benefits to commercial users of small UAVs.

\section{Conclusion}

The development of advanced small UAVs is hampered by a number of technology gaps. These include insufficient analysis methods for low Reynolds number aerodynamics, combined battery-motor-propeller propulsion, control effectiveness estimation, and weight estimation. Significant technology gaps also exist in individual subsystem technologies such as reliable, EMI/RFI resistant avionics/electronics, low-cost autonavigation, and high capacity/low weight battery technology. Unless a significant research effort is undertaken across a broad front, smaller UAVs will continue to have limited application. 